\title{
A REVIEW OF THE MONITORING AND MANAGEMENT OF SPOTTED-WING DROSOPHILA (DROSOPHILA SUZUKII) IN LOWBUSH BLUEBERRIES
}

\author{
CAROLYN WILSON \\ Dalhousie University, Faculty of Agriculture, \\ Truro, NS B2N $5 E 3$
}

\begin{abstract}
The recent arrival of spotted-wing drosophilia (Drosophila suzukii) to eastern Canada is a major threat to the million dollar lowbush blueberry industry. The highly fecund female fly lays her eggs in ripe soft-skinned fruit. The maturing larvae consume the fruit interior, ultimately resulting in fruit collapse and decreased yield. Effective monitoring and management of this pest is essential for reducing the risk of export market closure and economic losses. In this literature review, an integrated pest management plan is developed that outlines current monitoring and management practices for the pest and considers preventative physical, chemical and biological controls.
\end{abstract}

\section{INTRODUCTION}

Spotted-wing drosophilia (Drosophila suzukii) or SWD has recently colonized and rapidly dispersed across North America and Europe, complicating the production of lowbush blueberries (Vaccinium angustifolium) and other susceptible fruit crops. To lay eggs, the female inserts her sharp, serrated ovipositor into ripe berries and fruit. The developing larvae consume the fruit interior, resulting in fruit collapse and yield loss for producers. Crop loss is intensified by the high fecundity and short generation time of $D$. suzukii and infection must be treated quickly and effectively to minimize damage and spread. Due to the near-immediate dissemination of SWD across the globe, researchers and producers are scrambling to comprehend the biology and ecology of this invasive and noxious pest. With the threat of export market closure and million dollar yield 
losses, wild blueberry producers are relying on researchers to find effective monitoring techniques and control measures for D. suzukii.

\section{Biology and Ecology of Drosophila suzukii}

Originally described in Japan, Drosophilia suzukii was first identified in the United States in 1980, in Hawaii (Kaneshiro 1983). By August 2008, SWD had colonized and infested strawberries and caneberries in mainland California (Lee et al. 2011b). In subsequent years, the fly spread northward and eastward, reaching Atlantic Canada in 2010 (Hauser 2011). At the same time, spotted-wing drosophila rapidly dispersed across Europe from Spain and Italy to France, Switzerland and Germany by 2011 (Calabria et al.2012). Although drosophilids are not well-adapted for long distance flight, D. suzukii is easily dispersed by regional wind currents and is passively transported by global trade (Kimura 1992; Calabria et al. 2012). High dispersal rates of $D$. suzukii presents a significant challenge for controlling the species.

Drosophila suzukii are small drosophilids (2 to $3 \mathrm{~mm}$ ) with distinguishing dark spots on the leading top edge of wings of male

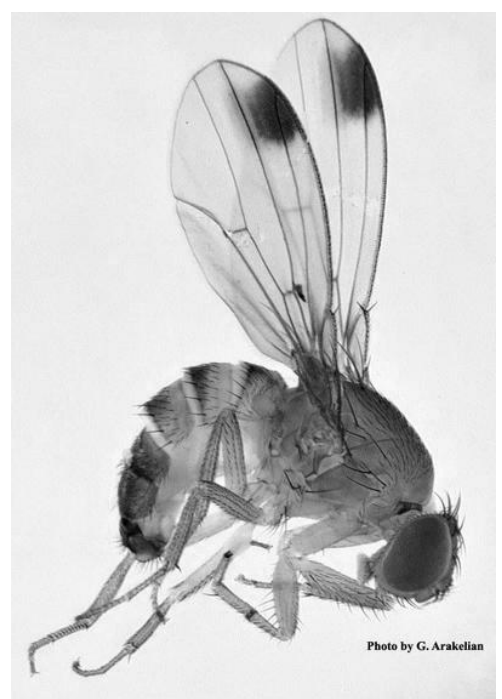

Fig 1 A magnified photograph of a male Drosophila suzukii. Image distinctly shows two dark markings on the tarsal segments of the forelegs and the black spots on the wings. Photo courtesy of G. Arakelian and the University of California, Center for Invasive Species Research. 
individuals, Fig 1. The females lack spots, but have a distinctly serrated or "saw-like" ovipositor on the abdomen (Cini et al. 2012). Both sexes of the species have red eyes, a brown-yellow thorax and continuous black stripes on the abdomen (Walsh et al. 2010). Although D. suzukii are generally dissimilar from other drosophilids, phenotypic characteristics are not static (males lacking spotted wings have been observed) and the only fully reliable identification tool is DNA barcoding (Cini et al. 2012).

A female lays her eggs in thin-skinned fruit by forming a slit in which she inserts 1 to 3 eggs (Lee et al. 2011a; Cini et al. 2012). Susceptible host fruits include a plethora of horticulturally significant crops: blackberries, blueberries, cherries, peaches, raspberries, strawberries, grapes (Lee et al. 2011a). Eggs hatch within 72 hours and the maturing larvae consume the fruit interior (Walsh et al.2010). Within two weeks, the larvae pupate. Shortly after emergence (1 to 5 days), Drosophila suzukii adults are able to produce viable progeny and over her lifetime, each female SWD oviposits an average of 380 eggs (Mitsui et al. 2006). At ideal temperatures (between 20 and $25^{\circ} \mathrm{C}$ ), populations of D. suzukii can repeat their entire developmental cycle in as little as 8 days (Walsh et al. 2010), causing exponential increases in the population and risk to the fruit industry.

Fruit damage caused by $D$. suzukii is the result of oviposition. Larval feeding of fruit interior compromises fruit integrity and reduces quality of pulp. After infection, fruit can pre-maturely drop from the stem or stored fruit may collapse post-harvest, ultimately reducing yield. In addition, the slit torn by the females' serrated ovipositor serves as an entry point for secondary bacterial or fungal infection, increasing the rate of fruit deterioration (Calabria et al.2012). Spottedwing drosophila has the potential to cause wide-spread damage to fruit crops across the Northern Hemisphere due to:

1. Wide host range for oviposition (Lee et al. 2011a, Walsh et al. 2010).

2. Rapid generation time.

3. Extreme fecundity (Walsh et al. 2010).

4. High dispersal potential via post-harvest fruit transportation and wind movements (Walsh et al. 2010).

5. Infection of ripe, pre-harvest fruit (Basoalto et al. 2013). 


\section{Eastern Canadian Lowbush Blueberry Production}

Lowbush or wild blueberries are woody perennials managed commercially to produce high yields of sweet, blue-colored fruit. Vaccinium angustifolium, low sweet, and Vaccinium myrtilloides, sourtop, are two species that naturally occur in eastern Canada and are well-adapted to the temperate climate (Kinsman 1993). When appropriate management practices are implemented, blueberry rhizomes can spread and plants densely cover acidic, abandoned woodland. Management of naturally occurring stands in eastern Canada and Maine led to commercial production of wild blueberries in the 1940s and 1950s. Since the 1950s, wild blueberries have been introduced to over 20 countries throughout the world (Barker et al. 1964, Kinsman 1993). Blueberries are highly marketable because they have high antioxidant levels and other health benefits - an important attribute in a progressively health-conscious consumer market (Kalt and Dufour 1997).

Wild blueberries are the major fruit export crop in Canada. Millions of tonnes of frozen berries are distributed to the United States, Japan, Germany and the Netherlands annually (Anonymous 2010). Production is limited to eastern Canada, with the majority occurring in Nova Scotia and New Brunswick. In these two provinces, there are over 1100 wild blueberry farms harvesting a combined marketed value of $\$ 42$ million in 2010 (Anonymous 2010). As markets continue to grow, Canadian producers are aiming to maximize crop yields and increase production acreage to feed increasing demand.

Commercial production of wild blueberries in eastern Canada is threatened by D. suzukii. Economic losses associated with the pest include reduced yield, and increased labor and chemical inputs costs for monitoring and management. Post-harvest fruit selection processes may be implemented, further increasing economic costs (Lee et al.2011b). Also, there is the potential for closures of export markets if/when importing countries impose thresholds of zero larvae in imported fruit and berries (Bruck et al. 2011). This would be a significant setback for Canadian lowbush blueberry producers, as over $75 \%$ of harvest is exported (Anonymous 2010).

The potential for economic damage as a result of SWD infection has been realized in several regions across the globe. Economic losses in Europe resulting from SWD infection include significant losses in Italy, 30-40\% loss in highbush blueberries, and France, $80 \%$ loss in strawberries (Lee et al. 2011b). Estimated yield losses in the US 
blueberry industry, based on 2008 production values, would result in \$56.7 million (US) in losses (Bolda et al. 2010). The economic impact of SWD is well shown: how can Nova Scotia wild blueberry producers minimize inevitable damage and loss?

\section{MONITORING AND MANAGEMENT OF SWD: AN INTEGRATED APPROACH}

Trapping and monitoring populations of SWD enables producers and researchers to quantify the severity of infection and evaluate the success of a control measure. Information obtained through pest monitoring can be compiled to map the distribution and spread of the species, as well as predict regions of future colonization (Burrack et al.2012). Researchers are currently optimizing trap design to actively and selectivity capture SWD adults (Basoalto et al. 2013, Walsh et al.2010). Post-harvest monitoring protocols for larval identification have also been developed, but have limited utility in a field setting and are used primarily in research.

D. suzukii adults are more frequently captured in colored traps with numerous entry holes baited with volatile fermented sugars.
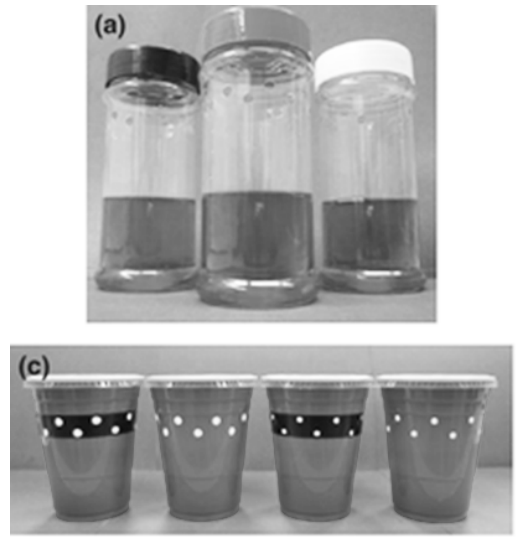
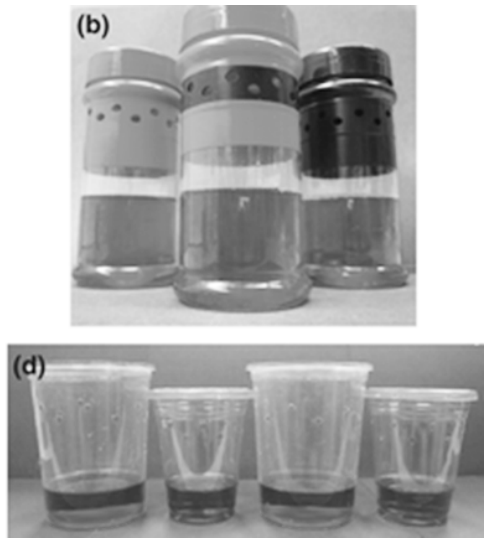

Fig 2 Cup-trap designs tested by Basoalto et al. (2013) and baited with apple cider vinegar to capture Drosophila suzukii adults: "(a) spice jars with black, red or white caps and ten 0.48 -cm holes, (b) all-red, all-black and 'Zorro' traps with ten $0.48-\mathrm{cm}$ holes, (c) $473-\mathrm{ml}$ red cups with or without a horizontal 1.5-cm black stripe and ten either $0.48-$ or $0.63-\mathrm{cm}$ holes and (d) $473-$ and 946-ml clear cups with 10 either 0.48- or 0.63-cm holes," (Basoalto et al. 2013). 
Basoalto et al. (2013) identified promising and commercially viable jar/cup-style designs after testing the attractiveness of several different trap designs to SWD, Fig 2 (2013). More flies were captured in $\mathrm{red} / \mathrm{black}$ striped traps with a greater number of entry areas (Basoalto et al. 2013, Lee et al. 2012). In SWD trapping studies, entry holes ranged in size from $0.32 \mathrm{~cm}$ to $0.63 \mathrm{~cm}$; smaller sized holes increased selectivity for drosophilids and decreased the number of non-target captures (Basoalto et al. 2013). Traps were typically placed above the crop canopy in shaded areas. Although females are responsible for the damage to fruit, they are difficult to distinguish from their drosophilid cousins. The distinguishing dark spot on the wings of males allow for easy identification of the species. It can be assumed that if males are present, females are too!

Apple cider vinegar replaced weekly is recommended for baiting, although a mixture of yeast-sugar-water has shown to be more effective (Walsh et al.2010). Overall, liquid baits are non-selective and difficult to handle in the field. The identification of selective chemical lures for SWD is an area of active research and some promising results using wine and vinegar volatiles have been observed by Cha et al. (2013). Researchers continue to design and evaluate trapping techniques for SWD in an effort to create a trapping protocol that is effective but affordable for producers.

\section{Cultural Control/Prevention}

Preventing infestation is the first step towards eradication of SWD. To reduce the exposure of fruit to D. suzukii, growers are encouraged to harvest ripe crops as early as possible. This strategy aims to disrupt crop-insect synchrony and eliminate niche accessibility. In addition, scrupulous sanitation of equipment and site can reduce habitat availability for flies as any fruit remaining in the field can provide a site for oviposition and feeding (Cini et al.2012). After harvest, rejected fruit should be treated or destroyed to kill any SWD life stages present (Walsh et al. 2010). Site sanitation is a major concern for lowbush blueberry producers. Depending on the field topography and the type of harvest equipment, upwards of $10 \%$ of fruit may remain in crop fields post-harvest. However, by properly adjusting and operating harvester equipment, fruit loss can be significantly reduced (Sibley 1993). 


\section{Physical Controls and Trapping}

Physical controls, such as netting or trapping, can be used to impede insect colonization. Netting (0.98 $\mathrm{mm}$ mesh) has successfully controlled SWD in highbush blueberry production (Lee et al.2011b), but is impractical for application in lowbush blueberries which are often developed on rough terrain with patchy field coverage. Use of mass-trapping to control SWD has been successful in China (Wu et al.2007). However, current bait is non-selective and applying this control to farms with large-acreage is costly and labour-intensive. After identifying SWD-specific pheromones and visual/auditory cues associating with courtship, researchers hope to develop synthetic compounds and trapping protocols that disrupt mating and oviposition (Cini et al. 2012).

\section{Chemical Controls}

Currently, chemical application is the most common method of SWD control applied by producers (Cini et al.2012). Use of insecticides is advantageous because it provides rapid and residual treatment of the invasive insect. In a recent American study, Bruck et al. (2011) demonstrated that synthetic pyrethroids, organophosphates and spinosyns provided 5-14 days of residual control of D. suzukii in lab and field replicates (2011). These insecticides have short pre-harvest intervals (less than 7 days), an important attribute of a pesticide applied to ripe fruit within days of harvest. Within these chemical groups there are several insecticides and formulations currently registered for emergency use in Canada and the United States. Chemical control thresholds for SWD are stringent and control application should be implemented after finding one SWD individual in a given field. SWD is an extremely noxious and invasive pest and without treatment, populations proliferate and cause significant economic damage.

Insecticide treatment is extremely lethal to SWD adults. However, there are several limitations to chemical control. As with most pesticides, frequent insecticide application increases selection pressures and the likelihood of insecticide resistance developing in pest populations. Using broad spectrum insecticides also kills beneficial species. Pesticide application can reduce populations of natural pollinators which are extremely important for the production of lowbush blueberries. Several other issues were observed by researchers studying chemical control of SWD: 
- Although specific insecticides may be registered for use in a given area, they may be restricted by international markets and government regulation. Compliance with maximum residue limits can significantly reduce control options. This is especially significant for fruit crops that rely on export markets, such as wild blueberries (Bruck et al. 2011).

- When populations of SWD were high, alternating use of insecticides had no significant effect on populations at harvest (Grassi and Pallaoro 2012).

- When studying pesticide efficacy, van Timmeren and Isaacs (2013) observed a significant decrease in insecticide efficiency when rain events occurred within days of application. This has significant implications for the lowbush blueberry industry, as Eastern Canada has a temperate climate.

\section{Biological Controls}

To find alternatives to chemical control, research is urgently needed to investigate viral pests, parasites and predators of SWD. Studies have identified parasites of D. suzukii in Japan, Europe and North America in field and laboratory conditions. One promising pupal ecto-parasite is Pachycrepoideus vindemmiae, studied in Oregon and British Colombia (Brown et al. 2011, Stacconi et al. 2013). When exposed to $P$. vindemmiae, only $11 \%$ of $D$. suzukii adults emerged from pupae, compared to an $85 \%$ emergence rate in uninfected control. In Summerland, British Columbia, the parasite was able to survive for 6 generations and led to the collapse of a colony of D. suzukii (Brown et al. 2011, Chabert et al. 2012). However, more research must be done to understand how/if these parasites are viable control measures in commercial fields.

After implementing a control, it is important to re-evaluate and continue to monitor pest populations to determine if further control measures are necessary. Integrated Pest Management programs are never static but rather they are in constant flux as superior control strategies are introduced and pest pressures fluctuate.

\section{CONCLUSION}

In conclusion, Drosophila suzukii is a noxious and invasive pest with the tenacity to damage the lowbush blueberry industry and production of other fruits in Canada. However, with proper monitoring 
and implementation of properly timed control measures, populations of this pest can be reduced and controlled. As researchers continue to study the biology and ecology of Drosophila suzukii, it is hopeful that new control measures and trapping strategies will be introduced in effort to save crops from this new threat.

\section{REFERENCES}

Anonymous. (2010). Statistical Overview of the Canadian Blueberry Industry, 2010. Government of Canada, Ottawa.

Barker, W.G., Hall, I.V., Aalders, L.E., \& Wood G.W. (1964). The lowbush blueberry industry in Eastern Canada. Economic Botany (18) 4:357365.

Basoalto, E., Hilton, R., \& Knight, A. (2013). Factors affecting the efficacy of a vinegar trap for Drosophila suzikii (Diptera; Drosophilidae). Journal of Applied Entomology 137: 561-570.

Bolda, M.P., Goodhue, R.E., \& Zalom, F.G. (2010). Spotted wing drosophila: potential economic impact of a newly established pest. Giannini Foundation of Agricultural Economics, University of California, Internal manuscript, p. 5-8.

Brown, P.H., Shearer, P.W., Miller, J.C., \& Thistlewood, H.M.A. (2011). The discovery and rearing of a parasitoid (Hymenoptera: Pteromalidae) associated with spotted wing drosophila, Drosophila suzukii, in Oregon and British Columbia. ESA annual meetings, Reno, USA.

Bruck, D.J., Bolda, M., Tanigoshi, L., Klick, J., Kleiber, J., DeFrancesco, J., Gerdeman, B., \& Spitler, H. (2011). Laboratory and field comparisons of insecticides to reduce infestation of Drosophila suzukii in berry crops. Pest Management Science 67 (11):1375-1385.

Burrack, H.J, Smith, J.P., Pfeiffer, D.G., Koeher, G., \& Laforest, J. (2012). Using volunteer-based networks to track Drosophila suzukii (Diptera: Drosophilidae) an invasive pest of fruit crops. Journal of Integrated Pest Management 3 (4):B1-B5.

Calabria, G., Máca, J., Bächli, G., Serra, L., \& Pascual, M. (2012). First records of the potential pest species Drosophila suzukii (Diptera: Drosophilidae) in Europe. Journal of Applied Entomology 136 (1-2):139-147.

Cha, D.H., Adams, T., Rogg, H., \& Landolt, P.J. (2012). Identification and field evaluation of fermentation volatiles from wine and vinegar that mediate attraction of spotted wing drosophila, Drosophila suzukii.Journal of Chemical Ecology 38 (11):1419-1431.

Chabert, S., Allemand, R., Poyet, M., Eslin, P., \& Gibert, P. (2012) . Ability of European parasitoids (Hymenoptera) to control a new invasive Asiatic pest, Drosophila suzukii. Biological Control 63 (1):40-47.

Cini, A., Ioriatti, C., \& Anfora, G. (2012). A review of the invasion of Drosophila suzukii in Europe and a draft research agenda for integrated pest management. Bulletin of Insectology 65 (1):149-160. 
Drummond, F., \& Yarborough, D. (2013). 210-Spotted Wing Drosophila: Pest Biology and IPM Recommendations for Wild Blueberries. University of Maine Extension. Accessed at umaine.edu/blueberries/ factsheets/insects/210-spotted-wing-drosophila/

Grassi, A., \& Pallaoro, M. (2012). Drosophila suzukii (Matsumura), a revolution for soft fruits in Trentino. In Ecofruit. 15th International Conference on Organic Fruit-Growing, Hohenheim, Germany.

Hauser, M. (2011). A historic account of the invasion of Drosophila suzukii (Matsumura) (Diptera: Drosophilidae) in the continental United States, with remarks on their identification. Pest Management Science 67 (11):1352-1357.

Kalt, W., \& Dufour, D. (1997). Health functionality of blueberries. HortTechnology 7 (3):216-221.

Kaneshiro, K.Y. (1983) Drosophila (Sophophora) suzukii (Matsumura). Proceedings of the Hawaiian Entomological Society 24:179.

Kimura, M.T. (1992). Tethered-flight activity of drosophilids (Diptera: Drosophilidae) in relation to habitat and resource distribution. Journal of Insect Behavior 5 (3):395-401.

Kimura, M.T. (2004). Cold and heat tolerance of drosophilid flies with reference to their latitudinal distributions. Oecologia 140: 442-449.

Kinsman, G. (1993). The history of the lowbush blueberry industry in Nova Scotia 1950-1990. Truro, Nova Scotia Department of Agriculture and Marketing.

Lee, J.C., Bruck, D.J., Curry, H., Edwards, D., Havilan, D.R., Van Steenwyk, R.A., \& Yorgey, B.M. (2011a). The susceptibility of small fruits and cherries to the spotted-wing drosophila, Drosophila suzukii. Pest Management Science 67 (11):1358-1367.

Lee, J.C., Bruck, D.J., Dreves, A.J., Ioriatti, C., Vogt, H., \& Baufeld, P. (2011b). In Focus: Spotted wing drosophila, Drosophila suzukii, across perspectives. Pest Management Science 67 (11):1349-1351.

Lee, J.C., Burrack, H.J., Barrantes, L.D., Beers, E.H., Dreves, A.J., Hamby, K.A., Haviland, D., Isaacs, R., Richardson, T., Shearer, P., Stanley, C., Walsh, D., Walton, V., Zalom, F., \& Bruck, D.J. (2012). Evaluation of monitoring traps for Drosophila suzukii (Diptera: Drosophilidae) in North America. Journal of Economic Entomology 105 (4): 1350-1357.

Mitsui, H., Takahashi, K.H., \& Kimura, M.T. (2006). Spatial distributions and clutch sizes of Drosophila species ovipositing on cherry fruits of different stages. Population Ecology 48:233-237.

Sibley, K.J. (1993). Effect of head-speed-ground-speed ratio on the picking effectiveness of a lowbush blueberry harvester. Canadian Agricultural Engineering 35 (1):33-39.

Stacconi, R., Grassi, M.V., Ioriatti, A.C., Dalton, D.T., Miller, B., Ouantar, M., Loni, A., Ioriatti, C., Walton, V.M., \& Anfora, G. (2013). First field records of Pachycrepoideus vindemiae as a parasitoid of Drosophila suzukii in European and Oregon small fruit production areas. Entomologia 1 (3):11-16. 
van Timmeren,S., \& Isaacs, R. (2013). Control of spotted wing drosophila, Drosophila suzukii, by specific insecticides and by conventional and organic crop protection programs. Crop Protection 54:126-133.

Walsh, D.B., Bolda, M.P., Goodhue, R.E., Dreves, A.J., Lee, J., Bruck, D.J., Walton, V.M., O'Neal, S.D., \& Zalom, F.G. (2010). Drosophila suzukii (Diptera:Drosophilidae): invasive pest of ripening soft fruit expanding its geographic range and damage potential. Journal of Integrated Pest Management 2:1-7.

Wu, S.R., Tai, H.K., Li, Z.Y., Wang, X., Yang, S.S., \& Sun, W. (2007). Field evaluation of different trapping methods of cherry fruit fly, Drosophila suzukii. Journal of Yunnan Agricultural University 22:776-778.

\section{APPENDIX}

Summative fact sheet for blueberry producers:

\section{Monitor and Manage Spotted-Wing Drosophilia}

\section{in Lowbush Blueberries}

\section{Why?}

Spotted-wing drosophila, SWD or Drosophila suzukii, is a vinegar fly that lays its eggs in ripe fruit and berries. Infection results in collapse of fruit, reducing harvestability and crop yield. SWD has the potential to cause million dollars in losses to the Canadian lowbush blueberry industry.

\section{How to Identify}

Adult flies are small, 2-3 $\mathrm{mm}$ in length, with red eyes. Male SWD have a distinct black spot (B) on the upper corner of each wing and two dark bands on their forelegs (C). The spot is absent in females who have a "saw-like" ovipositor for egg laying (D).

In the field, traps are examined for male individuals. Although females are present, they are more difficult to distinguish from non-SWD vinegar flies without a microscope.

\section{How to Monitor}

TRAP: Strip a piece of black tape around a 12 or 16 oz. covered red cup (Solo Co.) or spice jar. Punch 8-10 holes in the cup through the tape $(\sim 0.30 \mathrm{~cm})$.

Place traps in crop fields by the end of July (or earlier if advised). To set a trap, screw an adjustable ring clamp to a wooden stake. Position the clamp so that trap (held in the clamp) is about $20 \mathrm{~cm}$ above the crop canopy. Place as many traps per hectare as economically feasible, with a large proportion of traps placed near wooded areas and the field exterior.

Bait: Place $150 \mathrm{ml}$ of bait in the cup and replace/check weekly. Use either apple cider vinegar (easier to use, less effective) or yeast-sugar-water mixture (more labor-intensive, more effective).

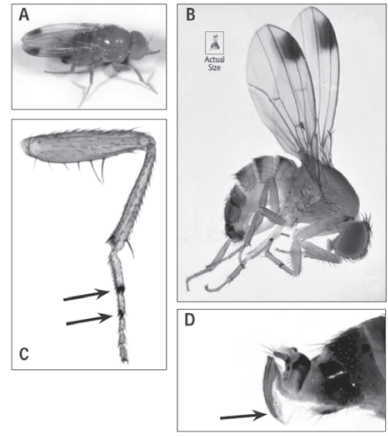

Photo above: Identification of spotted wing drosophila adult flies. A: adult flies, B: male adult with distinctive spotted wings, $C$ : dark bands of male tarsi, D: serrate ovipositor of female SWD. Photos from Michigan State University Extension.

Photo below: Trap for SWD in lowbush blueberries, UMaine.

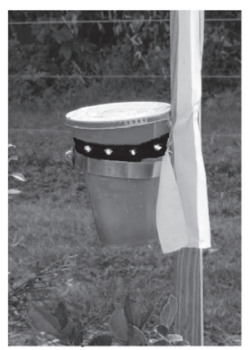




\section{Thresholds}

SWD is an extremely noxious and invasive pest with high dispersal potential. Currently, it is recommended to implement control measures after the first male is identified in a trap.

\section{How to Control}

\section{Preventing Infection}

Removing habitats for over-wintering adults and reducing egg-laying sites can reduce risk of SWD infection and re-infection. Some tips:

- Properly adjust and operate mechanical harvesters to minimize the number of berries that fall to the ground during harvest

- Remove used/rejected blueberry boxes from field site

- Harvest ripe blueberries as early and as quickly as possible

\section{Chemical Control Options}

Chemical should be applied as soon as one male adult is trapped or one female adult is identified microscopically. Comply with processor and export market pesticide restrictions and maximum residue limits. Be aware of issues with insecticide use including the development of insecticide resistance and possible damaging effect on natural populations, including beneficial species and pollinators.

\section{Other Control Options}

Researchers are currently developing and improving control protocols. Keep up to date with findings and new products available on the market.

\section{Evaluation}

After application, continue monitoring for SWD. Re-implement controls as necessary.

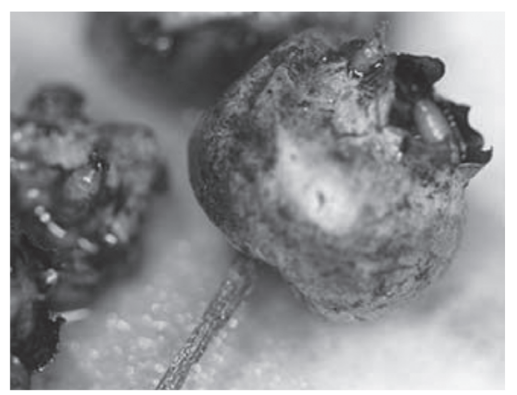

SWD pupation on the surface of blueberries. British Columbia Ministry of Agriculture. 